\title{
LECTURE CROISÉE
}

Étudier les dimensions sociopolitiques du droit

Jonathan Miaz

Presses de Sciences Po (P.F.N.S.P.) | «Gouvernement et action publique »

$2017 / 2 \mathrm{~N}^{\circ} 2$ | pages 131 à 140

ISSN 2260-0965

ISBN 9782724634969

Article disponible en ligne à l'adresse :

http://www.cairn.info/revue-gouvernement-et-action-publique-2017-2-page-131.htm

\section{Pour citer cet article :}

Jonathan Miaz, « Lecture croisée. Étudier les dimensions sociopolitiques du droit », Gouvernement et action publique 2017/2 ( $\left.{ }^{\circ} 2\right)$, p. 131-140.

DOI 10.3917/gap.172.0131

Distribution électronique Cairn.info pour Presses de Sciences Po (P.F.N.S.P.).

(C) Presses de Sciences Po (P.F.N.S.P.). Tous droits réservés pour tous pays.

La reproduction ou représentation de cet article, notamment par photocopie, n'est autorisée que dans les limites des conditions générales d'utilisation du site ou, le cas échéant, des conditions générales de la licence souscrite par votre établissement. Toute autre reproduction ou représentation, en tout ou partie, sous quelque forme et de quelque manière que ce soit, est interdite sauf accord préalable et écrit de l'éditeur, en dehors des cas prévus par la législation en vigueur en France. Il est précisé que son stockage dans une base de données est également interdit. 


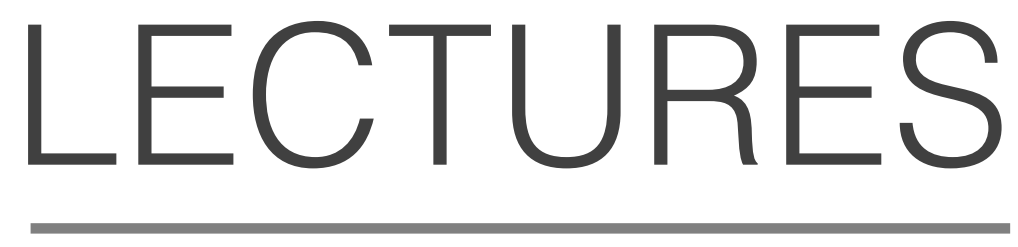

Lecture croisée

Comptes rendus 


\section{LECTURE CROISÉE}

\section{Étudier les dimensions sociopolitiques du droit}

\section{Thierry Delpeuch, Laurence Dumoulin, Claire de Galembert (2014)}

Sociologie du droit et de la justice

Paris, Armand Colin

\section{Mauricio Garcia Villegas (2015)}

Les Pouvoirs du droit : analyse comparée d'études sociopolitiques du droit

Issy-les-Moulineaux, LGDJ-Lextenso Éditions

e droit comme objet des sciences sociales connaît un intérêt croissant depuis plusieurs années en France ${ }^{1}$. Parus à quelques mois d'écart, les ouvrages discutés ici témoignent de cette évolution et contribuent à certifier « de l'existence académique d'un domaine de recherche [et à] la promotion du territoire d'une spécialisation ${ }^{2}$ ». Tous deux soulignent « la nature éminemment sociale du droit ${ }^{3}$ " et soutiennent l'idée fondamentale selon laquelle celui-ci ne peut être compris en dehors de sa dimension sociopolitique ${ }^{4}$.

Cette lecture croisée mettra en évidence que la sociologie du droit se caractérise par une interdisciplinarité et par une diversité d'approches et d'objets, redevables des histoires différenciées de la construction de ce domaine de recherche aux États-Unis et en France notamment. Nous reviendrons d'abord, à partir de l'ouvrage de M. Garcia $V$ illegas ${ }^{5}$, sur le développement de la sociologie du droit dans ces deux pays. Nous aborderons ensuite plus spécifiquement les travaux qui portent sur les relations entre le droit, l'État et l'action publique à partir du livre de T. Delpeuch, L. Dumoulin et C. de Galembert $^{6}$. II s'agira ainsi de souligner combien le droit en tant qu'objet des sciences sociales présente un grand intérêt heuristique pour analyser les sociétés, leur fonctionnement et leurs transformations ${ }^{7}$.

Dans Les Pouvoirs du droit, M. Garcia Villegas présente une analyse comparée et transdisciplinaire des idées sur le rapport entre droit, pouvoir politique et société dans trois régions du monde (États-Unis, France et Amérique latine) $)^{8}$. L'auteur souligne l'hétérogénéité des perspectives de

1. L. Israël (2013), "Legalise It! The Rising Place of Law in French Sociology ", International Journal of Law in Context, 9 (2), p. 262-278.

2. J. Commaille, "Préface ", dans T. Delpeuch, L. Dumoulin, C. de Galembert (2014), Sociologie du droit et de la justice, Paris, Armand Colin, p. 7.

3. T. Delpeuch, L. Dumoulin, C. de Galembert (2014), Sociologie du droit et de la justice, Paris, Armand Colin, p. 10.

4. Il faut souligner la parution, la même année, de l'ouvrage de Jacques Commaille qui montre bien, lui aussi, que le droit est indissociable du social, qu'il en est à la fois le produit et le producteur, et qu'il permet de révéler les modes de régulation sociale et politique des sociétés ainsi que leurs reconfigurations. J. Commaille (2015), À quoi nous sert le droit ?, Paris, Gallimard, p. 381.

5. Institut d'études politiques et relations internationales de l'Université nationale de Colombie.

6. Respectivement au Centre Marc Bloch de Berlin et à l'Institut des sciences sociales du politique, ENS Cachan.

7. J. Commaille, "Préface", dans T. Delpeuch, L. Dumoulin, C. de Galembert (2014), op. cit., p. 7.

8. II faut relever l'imposante bibliographie (71 pages) de cet ouvrage qui témoigne d'une fine connaissance de la littérature et qui permet aux lectrices et aux lecteurs qui le souhaitent d'aller plus loin à travers de très nombreuses références. 
sciences sociales et juridiques portant sur le droit, et propose de les regrouper sous le label général des « études sociopolitiques du droit » (ESD). À travers l'analyse de ce champ d'études transversal entre la sociologie du droit, la théorie critique du droit et les études sociojuridiques, M. Garcia Villegas montre que la situation des études sociopolitiques du droit en France, aux États-Unis et en Amérique latine dépend de facteurs d'ordres matériels et culturels tels que les rapports de pouvoir entre les acteurs du champ juridique, les conceptions sur le rôle du droit dans le pouvoir politique et la plus ou moins grande autonomie du savoir juridique à l'égard des sciences sociales.

Le manuel Sociologie du droit et de la justice nous permet d'entrer davantage dans les problématiques de ces recherches à partir d'une littérature internationale et interdisciplinaire. Ce faisant, ce livre "nous dit des choses essentielles sur les transformations de l'État et de ses modes d'agir, sur la redéfinition de sa place dans le cadre d'un processus historique de supranationalisation, sur les transformations de la régulation sociale et politique des sociétés ${ }^{9}$ ». T. Delpeuch, L. Dumoulin et C. de Galembert organisent leur propos autour de thématiques centrales dans la sociologie du droit et de la justice, de la conscience du droit des acteurs sociaux aux institutions judiciaires en passant par les relations entre le droit et l'État, l'action publique ou encore l'économie. En tant qu'il fait la synthèse des recherches portant sur ces différentes thématiques et de leurs apports, cet ouvrage constitue une référence de base essentielle en sociologie du droit.
La lecture de ces deux ouvrages est complémentaire, dans la mesure où ils adoptent une entrée différente pour dresser un panorama des travaux de sociologie du droit. Dans Les Pouvoirs du droit $^{10}$, M. Garcia Villegas fait porter son analyse sur l'histoire et l'évolution des idées, des courants et des débats qui animent ce domaine d'études. Dans Sociologie du droit et de la justice ${ }^{11}$, T. Delpeuch, L. Dumoulin et C. de Galembert offrent une vision d'ensemble des recherches en sociologie du droit à partir des problématiques et des thématiques sur lesquelles elles portent. À notre sens, l'intérêt de croiser ces deux ouvrages réside dans cette complémentarité qui permettra aux lectrices et aux lecteurs d'approfondir tant l'histoire de la constitution d'un domaine de recherche, que les différentes thématiques que ce dernier recouvre. En définitive, il apparaît que la sociologie du droit se caractérise par une internationalisation de travaux qui sont les produits d'histoires différenciées et dont la mise en dialogue se révèle féconde pour mieux saisir les objets de ce domaine d'étude, comme le sont l'État et l'action publique.

\section{Droit et sciences sociales aux États- Unis et en France : retour sur une évo- lution différenciée}

Comme le rappelle M. Garcia Villegas, pour les auteurs «fondateurs » de la sociologie tels qu'Émile Durkheim, Max Weber ou Karl Marx, le droit était étroitement lié à la réalité sociale et au pouvoir politique. Toutefois, après la Deuxième Guerre mondiale, la sociologie et la science politique exclurent le droit de leurs objets d'analyse

9. J. Commaille, "Préface ", dans T. Delpeuch et al. (2014), op. cit., p. 8.

10. M. Garcia Villegas (2015), Les Pouvoirs du droit : analyse comparée d'études sociopolitiques du droit, Issyles-Moulineaux, LGDJ-Lextenso Éditions.

11. T. Delpeuch, L. Dumoulin, C. de Galembert (2014), op. cit. 
dans une quête d'autonomie disciplinaire vis-à-vis de la science juridique et des facultés de droit ${ }^{12}$. Les rapports entre les sciences sociales et le droit ont donc d'abord été des relations de rejet ${ }^{13}$. Cette désaffection des sciences sociales à l'égard de l'objet juridique doit être différenciée d'un contexte à l'autre. Cette section s'efforce de rendre compte, à partir du livre Les Pouvoirs du droit, de cette évolution différenciée du domaine des études sociopolitiques du droit aux États-Unis et en France.

Aux États-Unis, les approches sociopolitiques du droit ont rencontré davantage de succès qu'en France, à la faveur d'une conception de la pratique juridique plus dynamique et politique. La dimension sociale du droit est en effet toujours présente, même parmi les juges et les praticiens, ce qui favorise le développement des études sociopolitiques du droit. Dans le champ juridique, la perspective antiformaliste $^{14} \mathrm{du}$ "réalisme juridique » a eu une influence considérable sur la culture juridique américaine ${ }^{15}$. En conséquence, selon M. Garcia Villegas, les études sociopolitiques du droit américaines sont marquées par le rejet de l'idée d'un droit conçu comme un savoir scientifique et par l'adoption d'une approche flexible et multidisciplinaire. Les sciences sociales y ont une influence certaine sur la pensée juridique et la dimension sociale du droit y est toujours présente ${ }^{16}$.

Dans les années 1960 et 1970, les études sociopolitiques du droit se développent dans la continuité du réalisme juridique autour de deux tendances. Premièrement, le mouvement académique Law and Society ${ }^{17}$ met l'accent sur l'analyse scientifique du droit à partir des sciences sociales. Créé en 1964, ce mouvement n'a cessé de croître non seulement aux États-Unis, mais aussi en Amérique latine, en Europe et en Asie. I repose sur quatre idées centrales: (1) l'intérêt pour la façon dont le droit influence la réalité sociale ; (2) I'utilisation des sciences sociales comme outils de connaissance de la réalité sociale pour pouvoir saisir la réalité du droit ; (3) l'adoption d'une conception politique progressiste et réformiste; (4) la reconnaissance de l'importance de l'analyse des processus institutionnels, en particulier les politiques publiques, pour parvenir aux objectifs de transformation politique proposés ${ }^{18}$. "La [Law and Society] a trouvé son identité dans la gestion, souvent difficile, de cette combinaison d'éléments: la recherche d'objectivité (science) et la défense des exclus (politique) ${ }^{19}$.

Deuxièmement, les Critical Legal Studies insistent davantage sur la critique politique du $\mathrm{droit}^{20}$. Ces études se rapprochent

12. M. Garcia Villegas (2015), op. cit. Sur les rapports entre science politique et science juridique, voir notamment : J. Chevallier (2004), "Science politique et science juridique », dans E. Darras, O. Philippe (dir.), La Science politique, une et multiple, Paris, L'Harmattan.

13. J. Commaille (2016), «À quoi nous sert le droit pour comprendre sociologiquement les incertidudes des sociétés contemporaines ? ", SociologieS [Online].

14. Au début du XX siècle, les professeurs de droit en France, en Allemagne et aux États-Unis étaient divisés sur la manière d'appréhender le phénomène juridique entre les « formalistes » qui mettent l'accent sur l'autonomie du droit, et les " antiformalistes ", qui postulent la sujétion et l'adaptation du droit à la réalité sociale.

15. M. Garcia Villegas (2015), op. cit., p. 110.

16. Ibid, p. 112.

17. Voir A. Vauchez (2001), "Entre droit et Sciences sociales. Retour sur l'histoire du mouvement Law and Society ", Genèses, 4 (45), p. 134-149.

18. M. Garcia Villegas (2015), op. cit., p. 181-182.

19. Ibid, p. 183-184.

20. Voir le chapitre VI. «Fenêtre : sociologie du droit » de A. Christin, E. Ollion (2012), La Sociologie aux États-Unis aujourd'hui, Paris, La Découverte, p. 93-106. 
des idées réalistes en s'intéressant au rôle du droit dans la société. " Cet intérêt se traduit, pour elles, dans trois réflexions qui portent sur : 1) les contradictions internes à la pensée juridique et l'impossibilité de les résoudre rationnellement, 2) la fonction politique de légitimation de cette pensée dans les sociétés capitalistes modernes, et 3 ) la possible transformation progressiste de la société à partir d'une conception politique de gauche du système juridique ${ }^{21}$. »

À partir des années 1990, on assiste à un renouvellement des études sociojuridiques aux États-Unis, à travers l'importante croissance du mouvement Law and Society et le développement des Legal Consciousness Studies ${ }^{22}$. Ces travaux affirment la nécessité de la recherche empirique afin de saisir le fonctionnement du droit dans la société et la manière dont il est utilisé par les acteurs. D'un point de vue théorique, ce courant repose sur une conception culturaliste et constructiviste de la réalité sociale où la dimension symbolique est un élément central. La réalité sociale est perçue comme étant construite à partir des représentations et des interprétations des acteurs sociaux. L'accent est donc mis sur l'aspect cognitif de la dimension symbolique du droit. Ces recherches insistent aussi sur la dimension constitutive du monde social.
M. Garcia Villegas évoque encore le développement de mouvements empiristes (Empirical Legal Studies et New Legal Realism) et la dissémination du mouvement critique à travers la Théorie Critique de la Race, la Théorie Féministe du Droit, ainsi que les débats sur les droits. Ces derniers portent sur l'utilisation croissante des droits dans les luttes politiques des mouvements sociaux et ont évolué selon deux axes de recherche. Un premier porte sur le rôle des avocats et des juristes qui défendent des intérêts sociaux ou des causes publiques (cause lawyers) ${ }^{23}$. Un deuxième axe de recherche porte sur les contextes dans lesquels les stratégies juridiques peuvent être politiquement rentables ${ }^{24}$. Dans ces débats sur les droits, il faut souligner l'importance du livre de Stuart Scheingold (1974), The Politics of Rights, qui présente le droit selon deux aspects : (1) comme un instrument de domination politique, dans la mesure où le «mythe des droits " (The Myth of Rights) constitue un système de croyances, une idéologie trompeuse qui assimile le droit à plus de justice sociale; (2) et comme un outil pour les luttes sociales, dans la mesure où les avocats peuvent stratégiquement enrôler les droits au profit de plus de justice sociale ${ }^{25}$ (The Politics of Rights) ${ }^{26}$.

En France, les visions sociopolitiques du droit ont mis plus de temps à émerger.

21. Ibid, p. 191.

22. Voir, par exemple S. Merry (1990), Getting Justice and Getting Even: Legal Consciousness among WorkingClass Americans, Chicago (III.), University of Chicago Press; P. Ewick, S. Silbey (1998), The Common Place of Law: Stories from Everyday Life, Chicago (III.), University of Chicago Press; J. Pélisse (2005), «A-t-on conscience du droit? Autour des "Legal Consciousness Studies" ", Genèses, 59 (2), p. 114-130.

23. A. Sarat, S. A. Scheingold (1998), Cause Lawyering. Political Commitments and Professional Responsibilities, Oxford, Oxford University Press; A. Sarat, S. A. Scheingold (2006), Cause Lawyers and Social Movements, Standford (Calif.), Standford University Press ; L. Israël (2001), " Usages militants du droit dans l'arène judiciaire : le cause lawyering", Droit et société, 3 (49), p. 793-824 ; L. Israël (2009), L'Arme du droit, Paris, Presses de Sciences Po.

24. M. McCann (1994), Rights at Work. Pay Equity Reform and the Politics of Legal Mobilization, Chicago (III.), University of Chicago Press.

25. T. Delpeuch et al. (2014), op. cit., p. 125-126 ; M. Garcia Villegas (2015), op. cit., p. 226.

26. P.-Y. Baudot, A. Revillard (dir.) (2014), "Politiques des droits ", Gouvernement et action publique, 4 (4); P.-Y. Baudot, A. Revillard (dir.) (2015), L'État des droits. Politique et pratiques des institutions, Paris, Presses de Sciences Po. 
Les droits ont été envisagés dans la tradition de Rousseau comme un attribut de l'État démocratique exprimant la volonté populaire à travers la loi. Dès lors, la loi a longtemps été considérée comme un facteur autonome et indépendant de la société, soit comme un instrument politiquement neutre. Ainsi, le droit y est longtemps resté un objet exclusif des juristes, les professeurs de droit préservant leur position hégémonique dans le champ juridique $^{27}$. La consolidation de la doctrine juridique, en France au cours du $x x^{e}$ siècle, et la prise de distance des sciences sociales par rapport au droit ont ainsi conduit à la prédominance du formalisme juridique. En Europe, dans les systèmes de droit civil, les critiques des antiformalistes sont externes à la doctrine juridique, politiquement plus radicales qu'aux États-Unis, et ne voient pas de possibilité d'émancipation sociale grâce au droit, dans la mesure où la doctrine juridique était conçue comme étant trop proche du pouvoir politique ${ }^{28}$.

La sociologie du droit naissante dans les années 1960 est dominée par les juristes issus des facultés de droit, alors qu'elle est aujourd'hui largement investie aussi par des sociologues. La sociologie du droit des juristes vise surtout à mobiliser les sciences sociales au service du droit. Le projet de sociologie législative de Jean Carbonnier va dans ce sens : la sociologie est utilisée comme un savoir destiné à améliorer la production de la loi. Dans les années 1970, on assiste aussi à la naissance du mouvement Critique du Droit qui visait à créer une nouvelle science du droit opposée à la doctrine juridique et qui le présente comme un appareil de domination politique ${ }^{29}$. Pierre Bourdieu s'intéresse lui aussi au droit pour en développer une explication sociologique compatible avec sa théorie des champs sociaux et pour dépasser la dichotomie entre vision internaliste et externaliste du droit ${ }^{30}$. À l'opposé, Bruno Latour développe une sociologie pragmatique du droit intéressée à l'action " en train de se faire ${ }^{31}$ ".

L'essor de la sociologie du droit en France est surtout le fruit du travail de plusieurs chercheurs formés en droit, en sociologie et/ou en science politique. Les deux ouvrages en discussion évoquent ainsi le rôle central joué par André-Jean Arnaud, Jacques Commaille, Pierre Lascoumes et Jacques Chevallier, et par la revue Droit et société, fondée en 1985. La sociologie du droit telle qu'elle se développe en France se caractérise par une ouverture disciplinaire, notamment sous l'impulsion de Jacques Commaille qui propose une sociologie politique du droit intéressée aux relations entre le juridique et le politique. Avec la montée d'une sociologie empirique, les travaux de sociologie du droit tendent à l'appréhender comme une activité sociale ${ }^{32}$ et à faire porter l'analyse sur ses usages sociaux et politiques - expression qui "permet de mettre en évidence les

27. P. Bourdieu (1986), "La force du droit », Actes de la recherche en sciences sociales, 64 (1), p. 3-19.

28. En Amérique latine, les études sociopolitiques du droit connaissent grosso modo la même évolution qu'en France. Elles sont rares, marginales et dispersées dans la région et à l'intérieur de chaque pays. Leur spécificité réside toutefois dans une importance plus grande accordée aux différences entre ce que disent les normes et ce qu'il se passe dans la réalité sociale.

29. M. Kaluszynski (2010), "Sous les pavés, le droit : le mouvement "Critique du droit" ou quand le droit retrouve la politique ", Droit et société, 76 (3), p. 523-541.

30. P. Bourdieu (1986), "La force du droit », op. cit.

31. B. Latour (2002), La Fabrique du droit. Une ethnographie du Conseil d'État, Paris, La Découverte.

32. P. Lascoumes, É. Serverin (1988), "Le droit comme activité sociale: pour une approche weberienne des activités sociales ", Droit et société, 9, p. 171-193. 
multiples façons dont les acteurs, professionnels ou citoyens se saisissent du droit en vue de produire des effets sociaux et politiques $^{33}$ " - et sur la portée sociale du droit $^{34}$.

Alors que, pendant longtemps en France, la sociologie du droit a été entravée par la culture juridique classique qui postule l'autonomie du droit, on assiste à un essor de ce domaine d'analyse. Max Travers parle à cet égard de «re-sociologisation de la sociologie du droit ${ }^{35}$ ". Ainsi, «la sociologie [du droit] participe d'un mouvement général de "sécularisation" du droit consistant à le rendre pensable non comme une instance en surplomb de la société mais comme une composante et un produit de celle-cis ${ }^{36}$.

\section{Droit, État et action publique : le droit pour saisir la régulation politique des sociétés}

Bien que l'on utilise le singulier pour parler de la sociologie du droit, cela renvoie en fait à une multiplicité de paradigmes sociologiques, de méthodes et de conceptions du droit. Les objets étudiés dans ce domaine de recherche sont également diversifiés, du rapport aux normes des individus à l'analyse des instances judiciaires et des interactions entre le droit et l'économie. Parmi ces thématiques, les relations entre le droit, l'État et l'action publique constituent des questionnements féconds explorés par la sociologie du droit.

Le droit constitue une forme particulière de norme au sein des multiples registres normatifs qui concourent à la régulation ${ }^{37}$ des activités sociales. La juridicisation désigne la montée en puissance de la régulation juridique. Cette notion "renvoie à la prolifération du droit, telle qu'observable à travers l'inflation législative et réglementaire, la multiplication des formes juridiques de règlement des rapports sociaux [...]. Considérée sous cet angle, la judiciarisation, en ce qu'elle évoque la montée en puissance des juges et des tribunaux, n'est qu'une expression de ce phénomène général $[\ldots]^{38} »$.

Si le social est saisi par le droit à travers les processus de juridicisation et de judiciarisation, il est lui-même, en retour, constitué socialement et politiquement. On peut dès lors faire porter l'analyse sur sa portée sociale, en le considérant comme une activité sociale. Ces perspectives permettent de souligner sa fonction sociopolitique qui invite à s'intéresser aux usages proprement politiques dont il fait l'objet et dont on peut identifier deux dimensions : interne, liée à la marge d'interprétation de ses opérateurs, et externe, en référence au droit comme instrument politique ouvert aux usages symboliques des textes juridiques ${ }^{39}$.

33. M. Garcia Villegas (2015), op. cit., p. 296.

34. L. Israël, G. Sacriste, A. Vauchez, L. Willemez (2005), Sur la portée sociale du droit. Usages et légitimité du registre juridique, Paris, PUF.

35. M. Travers (1993), "Putting Sociology Back into the Sociology of Law ", Journal of Law and Society, 20, p. 438-451, cité dans T. Delpeuch et al. (2014), op. cit., p. 20.

36. T. Delpeuch, L. Dumoulin, C. de Galembert (2014), op. cit., p. 13.

37. La notion de régulation est définie comme "ensemble des structures et des processus grâce auxquels un système social parvient à maintenir sa cohésion, à réaliser ses objectifs et à se pérenniser en dépit des perturbations internes et externes qui affectent son fonctionnement ». T. Delpeuch, L. Dumoulin, C. de Galembert (2014), op. cit., p. 40-41.

38. T. Delpeuch et al. (2014), op. cit., p. 44.

39. M. Garcia Villegas (2015), op. cit., p. 47. 
Cette dimension politique du droit invite à s'interroger sur les relations entre le droit, l'État et l'action publique. Le droit occupe en effet une place centrale dans le fonctionnement de l'État qui s'est construit en tant que mode de domination légal-rationnel, disposant du monopole de la violence légitime, légitimité qui repose sur la croyance partagée dans les vertus du droit à organiser les relations entre gouvernants et gouvernés ${ }^{40}$. T. Delpeuch, L. Dumoulin et $C$. de Galembert soulignent ainsi que "ce qui caractérise l'ordre étatique c'est donc le passage d'un mode de commandement fondé sur un rapport d'adhésion personnel ou sur des fondements traditionnels et ou religieux à une modalité plus abstraite, médiatisée aussi bien par la règle de droit que par les prescriptions réglementaires d'un appareil administratif ou judiciaire en charge de leur application ${ }^{41} »$. On peut parler d'un double mouvement de juridicisation de l'État et d'étatisation du droit, ou d'une "forme de réciprocité fonctionnelle ${ }^{42}$ » entre droit et État: l'État étant garant de la règle de droit, lequel l'habilite à avoir recours à la force. Enfin, le droit est aussi un principe organisateur et légitimateur du jeu politique.

Si le droit est ainsi un vecteur de la construction de l'État, il constitue aussi un instrument important de son action ${ }^{43}$. Quelle est dès lors sa place dans l'élaboration et la mise en œuvre de l'action publique? Les travaux qui se sont intéressés à la fabrique de la loi et à la production de l'action publique ont bien montré l'imbrication entre sphère politico-administrative, juridique et société, de même que l'hétérogénéité des acteurs, des arènes et des instances impliquées dans l'élaboration des lois (mobilisations, groupes d'intérêts, réseaux d'action publique, administration, Parlement, etc.). En considérant toutefois le droit comme une activité sociale et en s'interrogeant sur ses usages, des recherches ont mis en évidence combien les acteurs de la mise en œuvre contribuent aussi à la production des politiques publiques et du droit à travers un processus de création normative continu ${ }^{44}$. À travers leur appropriation des normes et leur pouvoir discrétionnaire, les street-level bureaucrats $^{45}$ peuvent ainsi infléchir le sens d'une politique publique. De leur côté, les destinataires de l'action publique sont aussi amenés à composer avec les règles, jusqu'à les remettre en question. Par leurs usages des règles et leurs actions stratégiques, ils peuvent influer sur les modes d'action des administrations.

À côté de son rôle dans la construction de l'État et comme instrument d'action publique, les travaux de sociologie du droit montrent que le droit peut aussi être un vecteur de transformations sociales et politiques. Les travaux portant sur les avocats engagés en faveur du libéralisme politique ${ }^{46}$

40. M. Weber (1995), Économie et société, tome 1 : Les Catégories de la sociologie, Paris, Pocket (1re éd. 1922).

41. T. Delpeuch, L. Dumoulin, C. de Galembert (2014), op. cit., p. 108.

42. F. Chazel (2009), "Communauté politique, État et droit dans la sociologie weberienne : grandeur et limites de l'entreprise ", L'Année sociologique, 59 (2), p. 290, cité dans T. Delpeuch et al. (2014), op. cit., p. 109.

43. Cf. P. Lascoumes, P. Le Galès (2004), Gouverner par les instruments, Paris, Presses de Sciences Po.

44. P. Lascoumes (1990), "Normes juridiques et mise en œuvre des politiques publiques », L'Année sociologique, 40, p. 43-71.

45. M. Lipsky (1980), Street-Level Bureaucracy : Dilemmas of the Individual in Public Services, New York (N. Y.), Russell Sage Foundation.

46. L. Karpik (1995), Les Avocats, entre l'État, le public, le marché. XIIP-XX' siècles, Paris, Gallimard, cité dans T. Delpeuch et al. (2014), op. cit., p. 119. 
et ceux sur les cause lawyers qui prennent appui sur le droit pour défendre une cause politique vont dans ce sens ${ }^{47}$. Les stratégies judiciaires des mobilisations sociales et les usages contestataires du droit soulignent donc combien le droit constitue à la fois une contrainte et une ressource pour les mouvements sociaux, ce qui en fait, dans une certaine mesure, des coproducteurs du droit. "Cette appropriation du droit par les acteurs sociaux se traduit par leur implication dans la production de normes juridiques, partant des normes d'action publiques [...] [ce] qui souligne le caractère illusoire de l'idée d'un grand partage entre un État que produirait le droit et une société qui s'y soumettrait ou chercherait à s'y soustraire ${ }^{48}$. " Finalement, Delpeuch et al. s'interrogent aussi, à partir des travaux qui portent sur les dynamiques d'internationalisation du droit - la construction européenne, l'importance croissante des droits de l'homme et des juridictions institutionnelles -, si le droit n'est pas en passe de devenir un vecteur de dépassement de l'État.

Cette lecture croisée montre au final la richesse autant que l'hétérogénéité des recherches et des approches de sciences sociales qui prennent le droit pour objet.
Les deux ouvrages le relèvent, la sociologie du droit se caractérise par son interdisciplinarité - dans la mesure où la sociologie dialogue avec la science politique, I'histoire, l'anthropologie et la science juridique - et par une importante internationalisation, lisible à plusieurs niveaux : la multiplicité des terrains nationaux, les phénomènes de transnationalisation, les évolutions régionales de ce domaine d'études et la circulation des travaux. Cette internationalisation, qui implique la mise en perspective de recherches produites dans différents contextes académiques, se révèle particulièrement féconde pour les analyses des objets de la sociologie du droit, comme l'État et l'action publique, ainsi que pour leur renouvellement. Pour conclure, comme l'écrit Jacques Commaille, « le droit est une entrée à forte valeur heuristique pour saisir le sens du fonctionnement des sociétés et de ce qu'elles deviennent ${ }^{49}$ ". Ces deux ouvrages arrivent donc à point nommé pour offrir un panorama des travaux existants dans un domaine d'études en expansion.

Jonathan Miaz

Université de Lausanne, Crapul, IEPHI

Université de Strasbourg, SAGE UMR7363 jonathan.miaz@gmail.com

47. L. Israël (2009), L'Arme du droit, Paris, Presses de Sciences Po.

48. T. Delpeuch, L. Dumoulin, C. de Galembert (2014), op. cit., p. 130.

49. J. Commaille, "Préface ", dans T. Delpeuch, L. Dumoulin, C. de Galembert (2014), op. cit., p. 7. 